\title{
Um Modelo para Gerenciamento de Trilhas em Ambientes de Computação Móvel
}

\author{
Jader Silva, João Rosa, Jorge Barbosa, \\ Laerte Franco \\ Universidade do Vale do Rio dos Sinos \\ \{jader,jrs, jbarbosa,laertef\}@unisinos.br
}

\author{
Débora N. F. Barbosa \\ Centro Universitário \\ La Salle \\ nice@unilasalle.edu.br
}

\author{
Luiz Antônio Moro Palazzo \\ Universidade Católica de \\ Pelotas \\ lpalazzo@ucpel.tche.br
}

\section{Resumo}

Nos ambientes de computação móvel, o acompanhamento da mobilidade permite a adaptação das aplicações aos contextos percorridos pelo usuário (ciência de contexto). Nos últimos anos, o uso conjunto de contextos $e$ perfis de usuários vem sendo considerado uma oportunidade para a distribuição de conteúdo. Além disso, o aprimoramento e a ampla adoção dos sistemas de localização vêm estimulando ainda mais o acompanhamento da mobilidade, viabilizando o uso de Trilhas. Uma trilha consiste no histórico dos contextos visitados por um usuário durante um periodo. Este artigo propõe um modelo para o gerenciamento de trilhas. Considera-se que a Ciência de Trilhas é uma evolução da proposta de uso conjunto de contextos e perfis. O texto apresenta um protótipo e sua aplicação em um ambiente educacional ubíquo para a distribuição de conteúdo guiada pelas trilhas de aprendizes.

\section{Introdução}

Nos últimos anos a evolução dos dispositivos móveis e das redes sem fio de alta velocidade vem impulsionando a adoção da Computação Móvel [18]. Além disso, o aprimoramento e a proliferação de Sistemas de Localização [8] vêm estimulando a adoção de soluções que considerem com precisão a localização dos usuários na prestação de serviços (Serviços Baseados em Localização [13]). Serviços mais aprimorados consideram informações de contexto, tornando a computação ciente do ambiente do usuário (Ciência do Contexto $[4,5]$ ). O uso conjunto de perfis de usuários e informações do ambiente vem sendo considerado uma oportunidade para a distribuição de conteúdo personalizado e contextualizado [2].

Recentemente, estudos mostraram que $o$ acompanhamento do usuário em sistemas de computação móvel com suporte à localização pode ser usado para o registro do histórico dos contextos visitados durante um período de tempo [21]. Esse registro normalmente recebe a denominação de Trilha $[6,11]$. As trilhas registram atividades de um usuário nos contextos percorridos, mantendo assim, um histórico de seus deslocamentos e de sua atuação em cada contexto. Esse artigo propõe um modelo para o gerenciamento de trilhas em ambientes de computação móvel, denominado UbiTrail. Nessa proposta, considera-se que a Ciência de Trilhas constitui uma evolução da proposta de uso conjunto de contextos e perfis de usuários [2].

Entre as áreas que deverão ser influenciadas pela exploração de trilhas, destaca-se a Educação Ubíqua $[14,16]$. No cenário educacional, a mobilidade do aprendiz suporta novas oportunidades de aprendizagem. O uso dos perfis de alunos em ambientes cientes de contexto [3] e o aperfeiçoamento automático desses perfis [12] são tópicos de pesquisa recentes. No entanto, a distribuição de conteúdo guiada pelas trilhas dos aprendizes é uma área de pesquisa ainda em aberto. Esse artigo apresenta um estudo de caso onde o UbiTrail foi integrado a um sistema de educação ubíqua (LOCAL [3]) e usado na distribuição contextualizada de objetos de aprendizagem.

O artigo está organizado em seis seções. A segunda seção descreve o modelo UbiTrail, considerando principalmente seus principais elementos e sua arquitetura. Por sua vez, a terceira seção aborda o uso do UbiTrail na computação móvel, mais especificamente na educação ubíqua. A seção quatro apresenta e avalia os resultados obtidos em um estudo de caso envolvendo o uso de trilhas na distribuição contextualizada de objetos de aprendizagem. A quinta seção discute os trabalhos relacionados, focando principalmente nas contribuições desse trabalho. A última seção apresenta conclusões e indica trabalhos futuros.

\section{Modelo UbiTrail}

\subsection{Conceitos Básicos}

O UbiTrail é um modelo dedicado ao gerenciamento de trilhas de Entidades. O termo entidade representa uma pessoa acessando recursos computacionais (smartphone, etc.) ou um objeto móvel (veículo, etc.). Podem ser gerenciadas trilhas relacionadas com qualquer entidade de 
interesse. Assim, diferentes aplicações para trilhas podem ser consideradas. Por exemplo, um veículo poderá ter uma trilha com todos os seus deslocamentos, registrando informações como as ocorrências de manutenção ou falhas.

Os registros relacionados aos contextos visitados pela entidade são armazenados em apenas uma trilha. A trilha é composta por uma sequência de registros de uma estrutura chamada ptrail (piece trail). As aplicações que utilizam o UbiTrail possuem internamente uma ptrail, composta pelos atributos: entidade, recurso, eventos, extensão e localização. Os valores dos atributos entidade e recurso são mantidos de forma estática na ptrail. Os valores dos atributos eventos, extensão e localização são atualizados conforme as ações ou deslocamentos realizados pela entidade e monitorados pela aplicação do UbiTrail.

O trailpoint é o processo que realiza a composição da trilha, enviando os valores que estão contidos na ptrail para a inserção do registro na trilha da entidade. O processo de trailpoint ocorre nas seguintes situações: (1) pela solicitação da entidade usando o seu dispositivo móvel; (2) quando a aplicação identifica automaticamente um evento realizado pela entidade, por exemplo, uma entrada ou saída em uma região. A Figura 1 exemplifica a criação da trilha de um usuário de dispositivo móvel que se desloca por quatro regiões. No trajeto, cada estrela indica um evento de formação da trilha. Cada evento ocasiona um trailpoint, ou seja, o envio da ptrail para registro de um ponto relevante na formação da trilha.

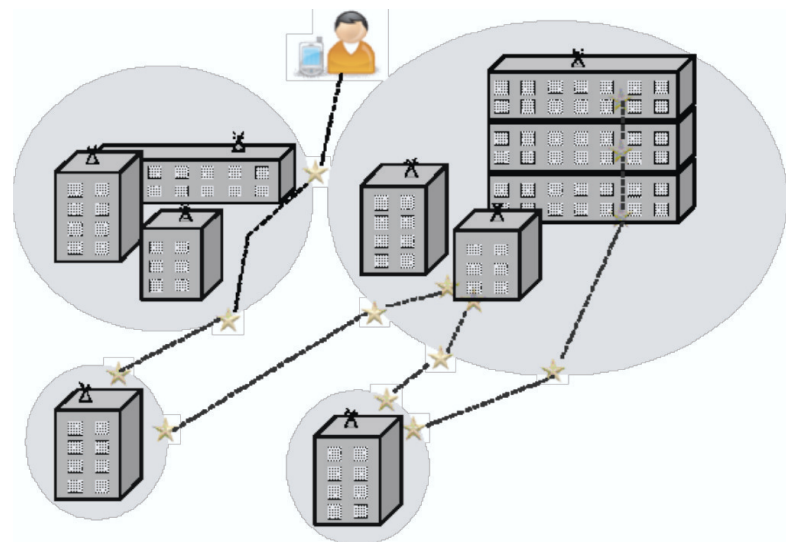

Figura 1. Exemplo de composição de uma trilha

\subsection{Arquitetura do Modelo}

O modelo está organizado em três partes: provedores externos, UbiTrailServer e UbiTrailClient. O UbiTrail considera a existência de dois provedores externos que fornecem informações de contexto e de localização. A Figura 2 apresenta a visão geral do modelo.

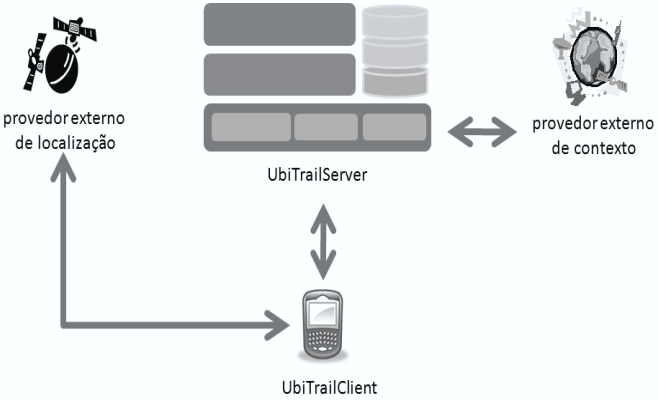

Figura 2. Visão geral do modelo

2.2.1. UbiTrailServer. O UbiTrailServer suporta os serviços usados pelas aplicações e também para o gerenciamento das trilhas, possuindo três camadas e três repositórios de dados (Figura 3a). A camada superior é formada pelo módulo TrailServices que gerencia os serviços fornecidos pelo servidor. Essa camada suporta duas categorias de serviços: básicos e especializados. Os serviços básicos são usados no gerenciamento das trilhas e os especializados suportam as funcionalidades específicas de uma aplicação. A camada intermediária consiste do módulo TrailContext que administra a comunicação com os provedores externos. A camada inferior (TrailCore) é composta pelos submódulos TrailManager, TrailRegister e TrailRepository. O TrailManager gerencia o fluxo de informações internas no UbiTrailserver e também as solicitações do UbiTrailClient. Uma das suas principais tarefas é o gerenciamento do processo de trailpoint. O TrailRegister autentica e manipula as entidades no UbiTrailserver. $\mathrm{O}$ TrailRepository disponibiliza uma interface para acesso às informações dos repositórios de dados. O acesso aos repositórios é necessário tanto para gerenciamento de informações de entidades como para a busca por definições na ontologia. O servidor possui ainda os repositórios contendo os dados das entidades, das trilhas e da ontologia. O repositório de ontologia armazena as regiões, localizações, eventos, entidades e recursos, seguindo a ontologia do UbiTrail [22]. O repositório de trilhas guarda as ptrails que formam as trilhas das entidades.

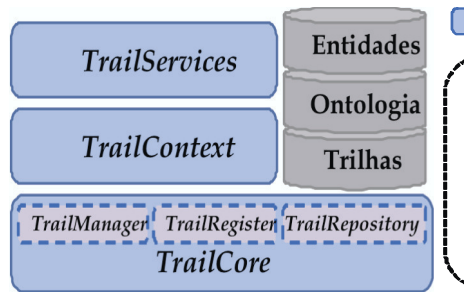

(a) UbiTrailServer

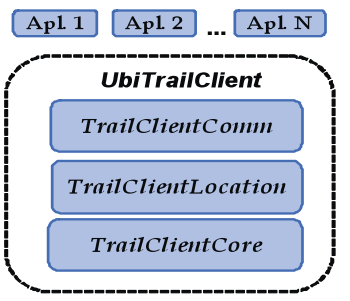

(b) UbiTrailClient
Figura 3. Arquitetura do UbiTrail 
2.2.2. UbiTrailClient. O UbiTrailClient reside no dispositivo móvel e suporta as aplicações do UbiTrail (Apl., Figura 3b). O cliente instancia e gerencia as ptrails das entidades, realizando o sincronismo das aplicações com o UbiTrailServer. O cliente está organizado em três camadas (Figura 3b). A camada superior trata da comunicação (TrailClientComm) do cliente com o servidor. A camada intermediária (TrailClientLocation) realiza a comunicação do dispositivo com o provedor externo de localização geográfica. A camada de gerenciamento (TrailClientCore) administra o acesso aos recursos do dispositivo.

\section{Uso de Trilhas na Computação Móvel}

O UbiTrail foi integrado ao sistema de Educação Ubíqua LOCAL (LOcation and Context Aware Learning) [3]. A distribuição de conteúdo depende da sua representação, seleção e disponibilização [2]. No LOCAL, a representação é baseada em metadados que categorizam os objetos segundo a norma IEEE/LOM [9]. A seleção é realizada pelo Tutor usando a localização e os perfis dos aprendizes e a disponibilização usa o sistema de comunicação.

\subsection{Serviços Especializados no UbiTrail}

A distribuição de conteúdo guiada por trilhas foi baseada em serviços especializados criados no UbiTrail e acessados pelo Tutor do LOCAL. A Tabela 1 resume os serviços criados. As informações retornadas pelos serviços são obtidas através da análise das trilhas dos aprendizes. As preferências são determinadas pela frequência de uso das mídias, dos dispositivos e dos aplicativos em um contexto.

Tabela 1. Serviços especializados

\begin{tabular}{|l|l|}
\hline \multicolumn{1}{|c|}{ Nome } & \multicolumn{1}{|c|}{ Descrição } \\
\hline PrefMidia & $\begin{array}{l}\text { Retorna uma lista com as mídias preferidas por um aprendiz } \\
\text { em um contexto (por exemplo, texto, vídeo ou áudio). }\end{array}$ \\
\hline PrefDispositivo & $\begin{array}{l}\text { Retorna uma lista com os dispositivos preferidos por um } \\
\text { aprendiz em um contexto (por exemplo, iPAQ ou desktop). }\end{array}$ \\
\hline PrefAplicativo & $\begin{array}{l}\text { Retorna uma lista com os aplicativos preferidos por um } \\
\text { aprendiz em um contexto (por exemplo, Adobe Reader ou } \\
\text { Windows Media Player). }\end{array}$ \\
\hline EscalonaOAs & $\begin{array}{l}\text { Retorna uma lista de sugestões de horários para acesso a um } \\
\text { OA para um aprendiz. }\end{array}$ \\
\hline OAsPreferidos & $\begin{array}{l}\text { Retorna uma lista com os OAs mais acessados em um } \\
\text { contexto por todos os aprendizes. }\end{array}$ \\
\hline
\end{tabular}

\subsection{Perfil Dinâmico e Contextualizado}

O modelo de perfil do LOCAL segue o padrão PAPI [15] acrescido dos estilos de aprendizagem propostos por Felder e Silverman [7]. As principais informações do perfil (Contato, Preferências, Interesses e Estilos) são estáticas e armazenadas no Assistente Pessoal (AP). Sendo assim, elas não mudam e acompanham o usuário independentemente do contexto visitado. A seção Contato armazena informações básicas do usuário (nome, endereço, e-mail e telefone). A seção Preferências armazena suas preferências relacionadas com dispositivos, aplicativos e os tipos de mídia (por exemplo, vídeo, áudio ou texto). As informações sobre Interesses seguem as áreas do conhecimento da ACM [1] e a seção Estilos contém seu estilo de aprendizagem [7]. O Tutor decide a distribuição de OAs para o aprendiz de acordo com seu perfil e considerando ainda os objetos disponíveis em cada contexto.

$\mathrm{Na}$ integração UbiTrail/LOCAL as trilhas são usadas para uma administração dinâmica e contextualizada do perfil. Usando os três primeiros serviços disponibilizados pelo UbiTrail (veja Tabela 1), o Tutor determina dinamicamente quais são as preferências do aprendiz no contexto que está sendo visitado. O histórico das visitas no contexto, registrado na trilha do aprendiz, permite a determinação de suas preferências contextualizadas e ainda permite que essas preferências sejam obtidas de forma atualizada, pois elas podem ter mudado na medida em que o aprendiz interagiu com os OAs. A integração do UbiTrail não substitui o perfil do aprendiz armazenado no AP do LOCAL. O Tutor usa as trilhas e o perfil conjuntamente, conforme descrito na próxima seção. Além disso, não é criada nenhuma nova estrutura de armazenamento para os perfis, pois os serviços do UbiTrail usam as trilhas armazenadas no repositório do UbiTrailServer para execução dos serviços especializados.

\subsection{Integração UbiTrail/LOCAL}

A integração UbiTrail/LOCAL é baseada nas seguintes alterações no LOCAL: (1) no Assistente Pessoal foi incluído o suporte para uso de serviços do UbiTrail; (2) as inferências realizadas pelo Tutor passam a considerar os perfis dos aprendizes e também os serviços disponibilizados pelo UbiTrail (descritos na Tabela 1). A Figura 4 mostra a dinâmica da integração. No LOCAL, os objetos de aprendizagem são disponibilizados para o aprendiz de acordo com as oportunidades que surgem durante o seu deslocamento pelos contextos. O Sistema de Localização informa ao Tutor o contexto onde está o aprendiz (passo 1). O Tutor usa essa informação, aliada ao perfil do aprendiz, para determinação dos objetos relevantes para o aprendiz e disponíveis no contexto (passo 2). Nessa etapa, o Tutor utiliza os serviços do UbiTrail para aperfeiçoamento de suas inferências. O Tutor notifica a disponibilidade dos objetos no contexto, através do Sistema de Comunicação (passo 3). O aprendiz recebe uma mensagem informando a disponibilidade (passo 4). Esse ciclo de distribuição de conteúdo contextualizado pode ser induzido por dois eventos: (1) a mudança de contexto do aprendiz ou (2) a inserção de novo material no repositório de objetos (neste caso, apenas os últimos três passos são executados).

Através da integração UbiTrail/LOCAL, o envio de OAs foi qualificado não apenas com o uso mais preciso de preferências, mas também com serviços específicos para a exploração de novas oportunidades pedagógicas que surgem através da disponibilidade de trilhas. Nesse sentido, 
destacam-se dois serviços. O serviço EscalonaOAs determina os períodos disponíveis para acesso aos OAs (por exemplo, o aprendiz se desloca todos os dias de trem entre Porto Alegre e São Leopoldo, antes e após as aulas na Unisinos). Através desse serviço, o Tutor aconselha o acesso a OAs nesses períodos. Por sua vez, o serviço OAsPreferidos permite ao Tutor a determinação de quais são os OAs mais acessados em um contexto, usando as trilhas de todos os aprendizes. Assim, o Tutor possui um parâmetro para indicação de um objeto de aprendizagem, filtrado através do seu uso em um contexto.

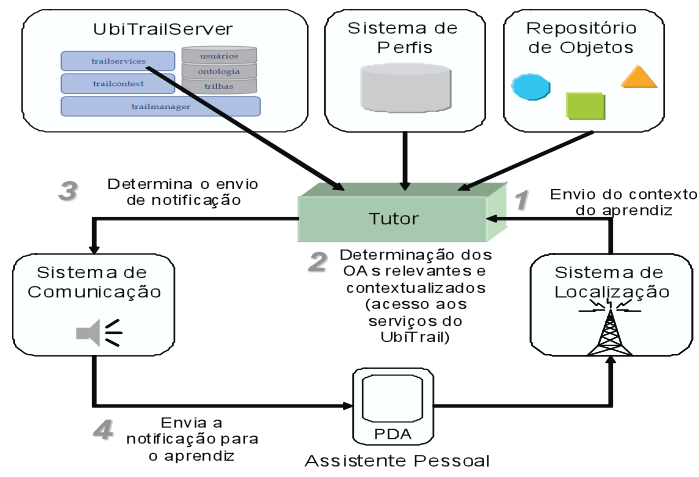

Figura 4. Distribuição de OAs guiada por trilhas

\subsection{Protótipos do UbiTrail e do LOCAL}

O protótipo do UbiTrail é organizado em pacotes. O pacote ubitrail.client representa o cliente. O ubitrail.server possui os subpacotes que representam as três camadas do servidor, o subpacote trail para controle das trilhas, o subpacote repository que suporta os três repositórios e o subpacote ontologies que contém as classes para o gerenciamento e manipulação da ontologia. O protótipo do UbiTrailServer foi implementado em Java. O framework JENA [10] foi usado para acesso a ontologia representada em OWL. Os serviços da camada TrailServices foram disponibilizados através de WebServices. O protótipo do UbiTrailClient foi implementado através da inserção de novos serviços no Assistente Pessoal (AP) do LOCAL.

O protótipo do LOCAL [3] foi implementado em C\#. O Tutor é um Windows Service e o AP foi desenvolvido com o .NET Compact Framework e executado em HP iPAQs 4700, munidos com Windows Mobile. Os demais componentes disponibilizam seus serviços para o Tutor e para o AP através de WebServices. A programação do suporte a redes sem fio usou a biblioteca OpenNETCF ${ }^{l}$.

A integração entre os protótipos foi principalmente baseada na alteração do Tutor para acesso aos serviços disponibilizados pelos WebServices do UbiTrailServices.

\footnotetext{
${ }^{1}$ OpenNETCF. http://www.opennetcf.com/
}

\section{Estudo de Caso}

\subsection{Obtenção de trilhas}

O estudo de caso foi baseado na geração simulada de trilhas de aprendizes, utilizadas no cenário real descrito no ambiente de validação (Seção 4.2). A opção pela simulação foi baseada nos seguintes fatores de interesse: (1) geração de trilhas para diversas regiões com ampla distribuição geográfica; (2) geração de trilhas que abrangessem no mínimo o intervalo de um período letivo do curso de Engenharia da Computação (EngComp) da Unisinos (aproximadamente 5 meses); (3) a impossibilidade de uso dos dispositivos móveis pelos alunos fora do campus. A Figura 5 apresenta o ambiente da simulação usado na geração de trilhas. $\mathrm{O}$ ambiente é composto por três regiões: Porto Alegre, Metrô e Unisinos.

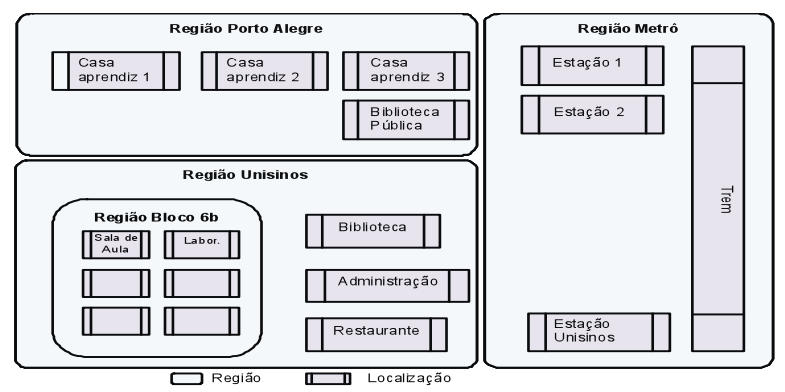

Figura 5. Mapa do ambiente de simulação

As regiões e localizações do ambiente são instâncias das classes RegionGeographical (região geográfica) e LocalizationGeographical (localização geográfica) da ontologia do UbiTrail [22]. Uma região pode ser composta por uma ou mais subregiões, mas ambos os casos são instâncias da classe região. Cada região ou subregião possui no mínimo uma localização com os valores de sua posição geográfica.

A região Porto Alegre suporta a localização das casas dos aprendizes e a biblioteca pública. A região Metrô representa a área onde os aprendizes se deslocam entre as suas residências e o campus da Unisinos. Essa região possui uma localização para cada uma das três estações e uma localização que representa a presença do aprendiz no trem. Sendo assim, é possível registrar os eventos realizados pelo aprendiz durante o deslocamento entre as estações. A terceira região corresponde ao campus da universidade. $\mathrm{Na}$ Unisinos, foi definida uma subregião para suporte as salas do Bloco 6B, onde está sediado o curso de EngComp. Essas salas foram organizadas em seis localizações, destacando-se a Sala de aula e o Laboratório. Foram cadastradas também as localizações Biblioteca, Administração e Restaurante.

A simulação envolveu cinco alunos da disciplina de Técnicas de Programação ${ }^{2}$ do segundo semestre de 2008 do

\footnotetext{
${ }^{2}$ http://www.inf.unisinos.br/ barbosa/grefe/pa2.htm
} 
curso de EngComp. As informações de perfil dos alunos foram cadastradas no LOCAL, focando suas preferências, seus possíveis estilos de aprendizagem e seus interesses relacionados com a disciplina em questão (usando as áreas de conhecimento da ACM [1]).

A geração das trilhas foi baseada nas possíveis atividades (eventos) realizadas pelos alunos durante o segundo semestre de 2008 (de 01/08/2008 a 23/12/2008). As trilhas foram geradas de forma simulada usando uma aplicação desenvolvida especificamente para esse fim, gerando os registros das trilhas em três etapas. Na primeira etapa foram gerados os registros de deslocamentos, que correspondem aos eventos de entrada e saída dos aprendizes nas localizações. A geração foi realizada para todos os alunos e baseada nos horários de aulas teóricas (presença na Sala de aula) e atividades práticas (presença no Laboratório). Os aprendizes João e Leonardo tiveram ainda registros de deslocamentos de suas casas (Casa aprendiz 1 e Casa aprendiz 2) até a Unisinos, passando pelas localizações relacionadas às estações de trem (Estação 1, Estação 2 e Estação Unisinos). Após a geração dos deslocamentos, na segunda etapa foram gerados os registros de utilização de dispositivos e aplicativos. Na terceira etapa foram gerados os registros de acesso aos objetos de aprendizagem, tendo como base o conteúdo previsto na disciplina de Técnicas de Programação.

A Tabela 2 mostra a quantidade de registros de eventos gerados durante a simulação. A Tabela 3 mostra um exemplo desses registros, os quais são usados na composição das trilhas.

Tabela 2. Quantidade de registros gerados na simulação

\begin{tabular}{|c|c|c|c|c|c|}
\hline Aprendiz & $\begin{array}{c}\text { Entrada/Saída } \\
\text { localização }\end{array}$ & $\begin{array}{c}\text { Login/Logoff } \\
\text { dispositivo }\end{array}$ & $\begin{array}{c}\text { Login/Logoff } \\
\text { aplicativo }\end{array}$ & $\begin{array}{c}\text { Acesso } \\
\text { OAs }\end{array}$ & Total \\
\hline João & 609 & 783 & 348 & 1479 & $\mathbf{3 2 1 9}$ \\
\hline Leonardo & 480 & 512 & 110 & 598 & $\mathbf{1 7 0 0}$ \\
\hline Vicente & 345 & 223 & 211 & 466 & $\mathbf{1 2 4 5}$ \\
\hline Rodrigo & 231 & 167 & 89 & 433 & $\mathbf{9 2 0}$ \\
\hline Douglas & 126 & 122 & 33 & 542 & $\mathbf{8 2 3}$ \\
\hline \multicolumn{7}{|r|r|r|}{ Total de Registros } & $\mathbf{7 9 0 7}$ \\
\hline
\end{tabular}

Tabela 3. Exemplos de registros

\begin{tabular}{|c|c|c|c|c|c|}
\hline Aprendiz & Disp. & Evento & Localização & $\begin{array}{l}\text { Extensão } \\
\end{array}$ & Data/Hora \\
\hline João & iPAQ & $\begin{array}{l}\text { Entrada em } \\
\text { localização }\end{array}$ & Sala de aula & & $\begin{array}{l}04 / 08 / 2008 \\
08: 01\end{array}$ \\
\hline Leonardo & $\mathrm{iPAQ}$ & $\begin{array}{l}\text { Entrada em } \\
\text { localizacão }\end{array}$ & Sala de aula & - & $\begin{array}{l}04 / 08 / 2008 \\
08: 12\end{array}$ \\
\hline Vicente & Desktop & $\begin{array}{l}\text { Entrada em } \\
\text { localizą̧ão }\end{array}$ & Laborató́rio & & $\begin{array}{l}06 / 108 / 2008 \\
14: 32 \\
\end{array}$ \\
\hline Vicente & $\begin{array}{l}\text { Desktop } \\
\end{array}$ & $\begin{array}{l}\text { Saida de } \\
\text { localização }\end{array}$ & Laboratório & - & $\begin{array}{l}06 / 08 / 2008 \\
15: 31\end{array}$ \\
\hline Vicente & Desktop & $\begin{array}{l}\text { Login em } \\
\text { dispositivo }\end{array}$ & Laboratório & - & $\begin{array}{l}07 / 08 / 2008 \\
16: 55\end{array}$ \\
\hline Rodrigo & Desktop & $\begin{array}{l}\text { Login em } \\
\text { aplicativo }\end{array}$ & Laboratório & Mídia Player & $\begin{array}{l}07 / 08 / 2008 \\
19: 22\end{array}$ \\
\hline Rodrigo & Desktop & $\begin{array}{l}\text { Logoff em } \\
\text { aplicativo }\end{array}$ & Laboratório & Midia Player & $\begin{array}{l}07 / 08 / 2008 \\
19 \cdot 33\end{array}$ \\
\hline Rodrigo & Desktop & $\begin{array}{l}\text { Logoff em } \\
\text { dispositivo }\end{array}$ & Laborató́rio & - & $\begin{array}{l}07 / 08 / 2008 \\
19: 55\end{array}$ \\
\hline João & iPAQ & Acesso OA & Sala de aula & $\begin{array}{l}\text { Arquivo: PDF } \\
\text { Midia: Texto } \\
\text { Tema: } \\
\text { Interfaces }\end{array}$ & $\begin{array}{l}\text { 18/08/2008 } \\
08: 25\end{array}$ \\
\hline Leonardo & Desktop & Acesso $\mathrm{OA}$ & Laboratório & $\begin{array}{l}\text { Arquivo: } \\
\text { MPEG } \\
\text { Mídia: Vídeo } \\
\text { Tema: Redes }\end{array}$ & $\begin{array}{l}18 / 08 / 2008 \\
14: 13\end{array}$ \\
\hline Vicente & $\mathrm{iPAQ}$ & Acesso OA & Laboratório & $\begin{array}{l}\text { Arquivo: MP3 } \\
\text { Mídia: Audio } \\
\text { Tema: Java }\end{array}$ & $\begin{array}{l}\text { 25/08/2008 } \\
07: 45\end{array}$ \\
\hline
\end{tabular}

\subsection{Ambiente de Validação}

A Figura 6 apresenta o mapa do segundo andar do Bloco 6B na Unisinos. Nesse andar estão sediados o MobiLab ${ }^{3}$ e o curso de graduação em Engenharia da Computação ${ }^{4}$. O cenário de validação é composto por nove salas, onde foram instaladas quatro antenas wireless Cisco Aironet 1100. O Sistema de Localização do LOCAL [17] é baseado na estratégia de triangularização de antenas wireless e permite a determinação da sala onde se encontra um usuário portando um dispositivo móvel. No experimento foram usados iPAQs 4700. Os equipamentos foram doados pela HP Computadores 5 .

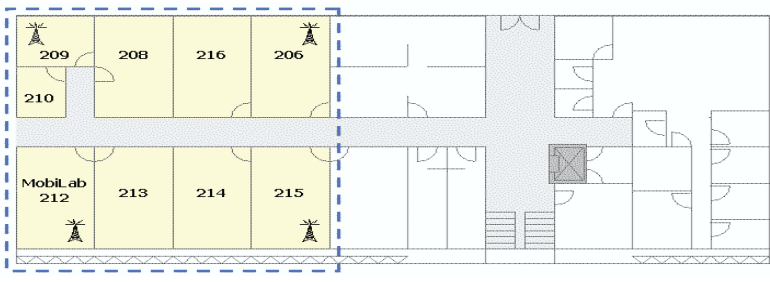

Figura 6. Disposição física do ambiente de validação

$\mathrm{Na}$ avaliação do protótipo UbiTrail/LOCAL, as salas mostradas na Figura 6 foram mapeadas para o ambiente de simulação apresentado na Figura 5. Assim foi criado um vínculo entre o ambiente real (Figura 6) e o ambiente de simulação (Figura 5), conforme representado na Figura 7. As salas 206 e 215 foram mapeadas para a representação das localizações Trem e Casa aprendiz 1. As demais salas foram consideradas as salas reais da Unisinos no Bloco 6B. Assim, durante a avaliação do protótipo, os deslocamentos entre algumas salas reais representavam deslocamentos virtuais. Por exemplo, um deslocamento da sala 214 (Sala de Aula) para a sala 206 (Trem) representava a presença do aprendiz no trem, se deslocando entre Porto Alegre e a Unisinos. A presença na representação virtual Trem, faz com que o Tutor inicie o processo de verificação de oportunidades pedagógicas, como se o aprendiz realmente estivesse fisicamente naquela localização.

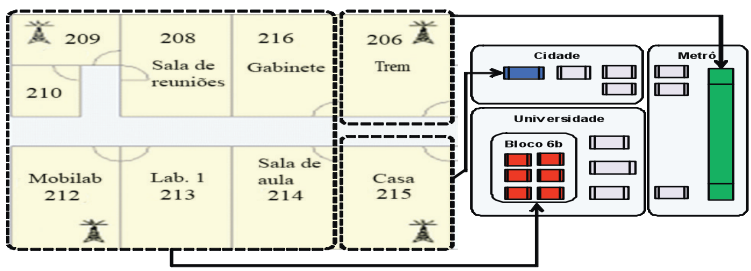

Figura 7. Cenário de validação

\footnotetext{
3 Laboratório de Pesquisa e Desenvolvimento em Computação Móvel http://www.inf.unisinos.br/ mobilab.

${ }^{4}$ Graduação de Referência em Engenharia da Computação. http://www.unisinos.br/graduacao/bacharelado/eng_comp.

${ }^{5}$ Prêmio recebido pelo MobiLab - "Grant HP Mobile Technology for Teaching 2005 - Latin American Region”.
} 


\subsection{Avaliação através de um Cenário}

Os cenários vêm sendo utilizados pela comunidade científica para avaliação de ambientes cientes de contexto (conforme abordagem de Dey [5]) e ambientes ubíquos (conforme Satyanarayanan [19]). Seguindo essa estratégia, foi criado um cenário para teste e avaliação da integração UbiTrail/LOCAL, envolvendo o cotidiano de um aluno do curso de Engenharia da Computação. Esse cenário enfoca a distribuição de OAs em diferentes contextos, com base nas trilhas desse aprendiz, conforme descrito a seguir:

"Jorge é o professor da disciplina de Técnicas de Programação do curso de Engenharia da Computação na Unisinos. No início da semana dedicada ao ensino básico de programação da linguagem Java, ele cadastra no repositório do LOCAL um conjunto de Objetos de Aprendizagem (OAs) que suportam diferentes preferências de mídias (textos, vídeos e áudios). O cadastramento envolve a indicação dos contextos onde os objetos devem ser disponibilizados. Na Sala de Aula é colocado um objeto curricular abordando conceitos básicos. No Laboratório estão objetos opcionais que poderão ser usados para aprimoramento, envolvendo diferentes temas de interesse (tais como, 'Interfaces' e 'Programação Distribuída'). João é um aluno do curso de Engenharia da Computação. No dia da aula de Técnicas de Programação, ao entrar na sala, João realiza o login no sistema LOCAL (veja tela na Figura 8a) e recebe a notificação de disponibilidade do OA curricular. João acessa o objeto e recebe um arquivo PDF (preferência texto). O serviço PrefMidia do UbiTrail foi usado pelo LOCAL para determinação dessa preferência, pois João já teve várias aulas e sempre usou objetos textuais. Os OAs de todas as preferências de mídia estão disponíveis, ou seja, João poderia ter acessado um objeto direcionado para outra preferência, fato que seria registrado na sua trilha e usado na determinação da preferência de mídia naquele contexto. Após a aula, João entra no laboratório e recebe o aviso de disponibilidade de um OA que aborda um tema de seu interesse em Java (por exemplo, 'Interfaces'). Esse objeto não foi cadastrado pelo professor, mas sim, reside continuamente no laboratório e vêm sendo usado por todos os alunos que têm interesse nesse tema. O LOCAL usou o serviço OAsPreferidos para indicação desse objeto. No laboratório, João prefere objetos de áudio, pois assim houve o conteúdo enquanto usa o computador (determinado pelo serviço PrefMidia). João acessa o objeto e, logo após seu uso, recebe outro aviso de disponibilidade de OA (nesse caso, um objeto opcional cadastrado pelo professor, veja tela na Figura 8b) que aborda seu tema de interesse. Sempre que o aluno recebe um aviso de OA, ele pode postergar o acesso. Essa ação indica que o aluno tem interesse no objeto, mas não quer acessá-lo naquele instante, mas sim na primeira oportunidade de tempo disponível. João posterga o acesso ao objeto opcional (tela na Figura 8c). No final do dia, João desloca-se para a estação de trem. Quando entra no trem, João é notificado pelo LOCAL que um OA postergado foi identificado. O LOCAL usa o serviço EscalonaOAs para definição desse envio. Ao chegar na sua residência, João recebe ainda a notificação que o professor disponibilizou um OA adicional para outro de seus temas de interesse, ou seja, 'Programação Distribuída'. No cadastro o professor informou que os alunos que tivessem interesse naquele tema deveriam ser notificados independentemente da sua localização."
O cenário foi testado no ambiente de validação descrito na Seção 4.2. O aluno João portando um iPAQ 4700 simulou o cenário, realizando os deslocamentos pelas salas do ambiente. O trem e a casa do aprendiz foram simulados através das salas 206 e 215. A Tabela 4 resume a sequência de ações, destacando o personagem envolvido.

Tabela 4. Dinâmica do cenário de validação

\begin{tabular}{|c|l|}
\hline Personagem & \multicolumn{1}{c|}{ Ação } \\
\hline Professor & $\begin{array}{l}\text { Cadastra os objetos de forma contextualizada no } \\
\text { LOCAL. }\end{array}$ \\
\hline Aprendiz & $\begin{array}{l}\text { Entra na sala de aula e realiza o login no LOCAL, } \\
\text { usando um iPAQ 4700. Veja tela na Figura 8a. }\end{array}$ \\
\hline $\begin{array}{l}\text { UbiTrail/ } \\
\text { LOCAL }\end{array}$ & $\begin{array}{l}\text { LOCAL detecta a entrada do aprendiz na sala de aula. } \\
\text { Tutor chama o serviço PrefMidia do UbiTrail para } \\
\text { determinação da preferência de mídia do aprendiz no } \\
\text { contexto. Logo após, solicita o envio de uma } \\
\text { mensagem informando a disponibilidade do OA na } \\
\text { mídia preferida. }\end{array}$ \\
\hline Aprendiz & Acessa o objeto curricular. \\
\hline Aprendiz & Desloca-se para o laboratório. \\
\hline UbiTrail/ & $\begin{array}{l}\text { LOCAL detecta a entrada do aprendiz no laboratório. } \\
\text { O Tutor usa o serviço OAsPreferidos do UbiTrail } \\
\text { para verificar a disponibilidade de OAs de interesse do } \\
\text { aluno que estejam sendo bastante acessados naquele } \\
\text { contexto. O Tutor usa ainda o serviço PrefMidia para } \\
\text { definção da mídia preferida pelo aprendiz naquele } \\
\text { contexto. O Tutor solicita o envio de mensagem } \\
\text { informando a disponibilidade de OA. }\end{array}$ \\
\hline Aprendiz acessa o objeto. \\
\hline Aprendiz
\end{tabular}

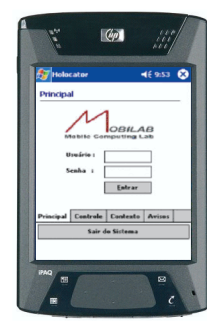

(a) Login no iPAQ 4700.

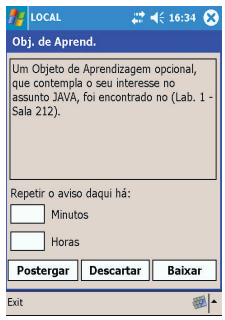

(b) Aviso de OA.

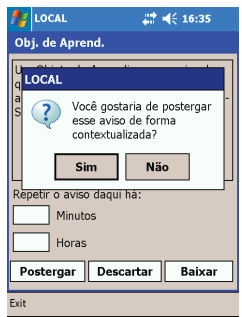

(c) Acesso postergado.
Figura 8. Telas do cenário 


\section{Trabalhos Relacionados}

O Hermes [6] é um framework para a criação de aplicações que envolvem o deslocamento de usuários durante a realização de atividades em diferentes localizações. No Hermes, uma trilha consiste em um trajeto sugerido para que o usuário cumpra as atividades de forma eficiente. A proposta não contempla o registro de atividades em contextos, conforme proposto pelo UbiTrail. O Hermes também não suporta a distribuição de conteúdo.

Levene e Peterson (Trail Records [11]) propõem a utilização de dispositivos móveis para registro de experiências de aprendizagem usando diferentes mídias (por exemplo, texto, vídeo e áudio) durante a visita em diferentes localizações. A sequência dos registros forma uma estrutura denominada trilha, a qual pode ser usada para recompor a experiência do aprendiz no trajeto visitado. Apesar de vincular conteúdo com localizações, não existe uma abordagem que suporte à distribuição de conteúdo guiada pelos contextos. Além disso, o modelo não é genérico, enfocando especificamente o registro de experiências com mídias.

O PELEP [12] propõe o aperfeiçoamento automático do perfil de aprendizes em ambientes de educação ubíqua. O modelo registra suas atividades durante um período de tempo e, tendo como base esse registro, atualiza periodicamente suas preferências. O PELEP não registra continuamente o histórico dos aprendizes em contextos, ou seja, não suporta o gerenciamento de trilhas. Além disso, o modelo enfoca especificamente a educação ubíqua.

O Life Annotation [20,21] propõe a anotação das localizações percorridas por usuários de dispositivos móveis, compondo uma trilha de locais visitados. Além disso, o modelo propõe que sejam registradas todas as informações possíveis relacionadas com cada local. O objetivo é o registro das atividade de um usuário, permitindo consultas no futuro. A proposta permite consultas genéricas relacionadas com as trilhas, mas não possui nenhum mecanismo que permita a distribuição de conteúdo. Além disso, Smith salienta como trabalho futuro de sua tese de doutorado [21] o uso de ontologias para padronização das informações de trilhas.

A tabela 5 sistematiza a comparação das propostas, incluindo o UbiTrail. A tabela é baseada nos seguintes critérios: (1) Histórico: indica se a proposta mantém o histórico de visitas a contextos; (2) Distribuição de conteúdo: indica a existência de mecanismo que suporte a distribuição de conteúdo baseado nas trilhas; (3) Registro padronizado: indica se é usada uma estratégia para padronização das informações de trilhas; (4) Geração automática: indica se as informações de trilhas são geradas de forma automática; (5) Registro de contexto: indica se a proposta registra informações de contexto nas trilhas; (6) Generalidade: indica se o modelo suporta diferentes aplicações.
Tendo como base a revisão bibliográfica desse artigo, pode-se afirmar que a integração UbiTrail/LOCAL cria o primeiro ambiente com suporte à distribuição de conteúdo guiado por trilhas. Além disso, entre os trabalhos pesquisados, o UbiTrail é o único que padroniza as informações com uma ontologia.

Tabela 5. Comparação das propostas

\begin{tabular}{|l|c|c|c|c|c|}
\hline Critério & Hermes & $\begin{array}{c}\text { Trail } \\
\text { Records }\end{array}$ & PELEP & $\begin{array}{c}\text { Life } \\
\text { Annotation }\end{array}$ & UbiTrail \\
\hline $\begin{array}{l}\text { Histórico de } \\
\text { contextos }\end{array}$ & Não & Sim & Não & Sim & Sim \\
\hline $\begin{array}{l}\text { Distribuição } \\
\text { de conteúdo }\end{array}$ & Não & Não & Sim & Não & Sim \\
\hline $\begin{array}{l}\text { Registro } \\
\text { padronizado }\end{array}$ & Sim & Não & Sim & Não & Sim \\
\hline $\begin{array}{l}\text { Geração } \\
\text { automática }\end{array}$ & Sim & Sim & Sim & Sim & Sim \\
\hline $\begin{array}{l}\text { Registro de } \\
\text { contexto }\end{array}$ & Não & Sim & Sim & Sim & Sim \\
\hline $\begin{array}{l}\text { Generalidade } \\
\text { do modelo }\end{array}$ & Sim & Não & Não & Sim & Sim \\
\hline
\end{tabular}

\section{Considerações Finais}

Este artigo propôs um modelo para o gerenciamento de trilhas e sua aplicação na distribuição de conteúdo em um ambiente de educação ubíqua. Embora o UbiTrail inicialmente tenha sido integrado ao LOCAL, sua proposta é genérica o bastante para ser aplicada em outros sistemas que possam usufruir de trilhas para seu aperfeiçoamento. $\mathrm{O}$ uso de trilhas permite aos sistemas de educação ubíqua uma atuação mais eficaz, pois podem ser usadas informações mais precisas do comportamento dos aprendizes.

As principais conclusões foram: (1) o uso de trilhas confirma o potencial da computação móvel para o aprimoramento da distribuição de conteúdo; (2) a disponibilidade do histórico de visitas aos contextos estimula ainda mais o uso de dispositivos móveis como instrumento para acesso aos conteúdos contextualizados; (3) o UbiTrail suporta a distribuição de conteúdo guiada por trilhas; (4) o protótipo e o experimento comprovaram a viabilidade da proposta.

O UbiTrail e sua integração ao LOCAL constituem uma proposta inicial. As seguintes atividades permitirão a continuidade do estudo: (1) realizar testes adicionais com as trilhas obtidas por simulação (por exemplo, os serviços PrefDispositivo e PrefAplicativo não foram usados); (2) aperfeiçoar a heurística para determinação das preferências nos contextos (frequência de uso); (3) avaliar o desempenho dos serviços envolvendo uma quantidade massiva de registros de trilhas; (4) realizar avaliação do modelo envolvendo o monitoramento de usuários para a geração de trilhas reais, durante um período de tempo que permitisse a avaliação dos seus comportamentos. 


\section{Referências Bibliográficas}

[1] ACM, "The 1998 ACM Computing Classification System", avalilable at http://www.acm.org/about/class/1998, 2009.

[2] J. L. V. Barbosa et al. "Distribuição de Conteúdo em Ambientes Conscientes de Contexto", Proc. 13th Brazilian Symposium on Multimedia and the Web, 2007, pp. 1-8.

[3] J. L. V. Babosa et al. "LOCAL: a Model Geared Towards Ubiquitous Learning", Proc. 39th SIGCSE Technical Symposium on Computer Science Education, ACM Press, Portland, USA, 2008, pp. 432-436.

[4] A. K. Dey, "Understanding and Using Context", Personal and Ubiquitous Computing, vol. 5, no. 1, 2001, pp. 4-7, doi: 10.1007/s007790170019.

[5] A. K. Dey, D. Salber, and G. D. Abowd, G.D. "A conceptual framework and a toolkit for supporting the rapid prototyping of context-aware application”. Human-Computer Interaction, vol.16, no.2, 2001, pp. 97-166.

[6] Driver, C. An Application Framework for Mobile, ContextAware Trails. PhD thesis, University of Dublin, 2007

[7] R. M. Felder and L. K. Silverman. "Learning and Teaching Styles in Engineering Education". Engineering Education, vol.78, no.7, 1988, pp. 674-681.

[8] J. Hightower, and G. Borriello, "Location Systems for Ubiquitous Computing", Computer, vol. 34, no. 8, 2001, pp. 57-66, doi: 10.1109/2.940014.

[9] IEEE/LTSC/LOM Learning Technology Standards Committee. "Draft Standard for Learning Object Metadata", available at http://ltsc.ieee.org/wg12/files, 2009.

[10] Jena. "A Semantic Web Framework for Java", available at http://jena.sourceforge.net, 2009.

[11] M. Levene, and D. Peterson. Trail Record and Ampliative Learning. Research Report BBKCS-02-01, School of Computer Science and Information Systems, University of London, 2002.

[12] D. Levis, J. L. V. Barbosa, et al. "Aperfeiçoamento Automático do Perfil do Aprendiz em Ambientes de Educação Ubíqua", Revista Brasileira de Informática na Educação, vol.16, no.1, 2008, pp.29-41.

[13] MobileIN Technologies, "Location Based Services (LBS)", http://www.mobilein.com/location_based_services.htm. 2009.

[14] H. Ogata, and Y. Yano, "How Ubiquitous Computing can support language learning", Proc. of KEST, 2003, pp. 1-6.

[15] PAPI - IEEE P1484.2/d7, "Draft Standard for Learning Technology - Public and Private Information for Learners", available at http://www.cen-ltso.net/main.aspx?put=230, 2009.

[16] Y. Rogers, S. Price, C. Randel, D.S. Fraser, M. Weal, and G. Fitzpatrick, "Ubi-learning Integrates Indoor and Outdoor Experiences", Communications of the ACM, vol. 48, no. 1, 2005, pp. 55-59, doi: 10.1145/1039539.1039570.

[17] C. R. Rolim, N. Sonntag and J. L. V. Barbosa, "HLS: Modelo para Desenvolvimento de Aplicações Sensíveis à Localização". XI Workshop em Sistemas Computacionais de Alto Desempenho, 2008, pp. 227-234.
[18] M. Satyanarayanan. "Fundamental challenges in mobile computting", Proc. 15th ACM Symposium on Principles of Distributed Computing, 1996, pp. 1-7, doi: 10.1145/248052.248053.

[19] M. Satyanarayanan. "Pervasive Computing: Vision and Challenges", IEEE Personal Communications, vol. 8, no. 4, 2001, pp. 10-17.

[20] A. D. Smith et al. "Towards Truly Ubiquitous Life Annotation", Memories for Life Colloquium 2006, The British Library, London, England, 2006.

[21] A. D. Smith. "Who Controls the Past Controls the Future Life Annotation in Principle and Practice", $\mathrm{PhD}$ thesis, University of Southampton, available at http://eprints.ecs.soton.ac.uk/16554/, 2008.

[22] J. M. Silva. "UbiTrail: Um Modelo para Gerenciamento de Trilhas Orientado a Ambientes Ubíquos", Dissertação (Mestrado em Computação Aplicada), Universidade do Vale do Rio dos Sinos, 2009. 